\section{Case Reports in Ophthalmology}

\title{
A Case of Rhegmatogenous Retinal Detachment at Late Stage following Endogenous Bacterial Endophthalmitis
}

\author{
Daisaku Kimura ${ }^{a, b}$ Takaki Sato $^{a}$ Hiroyuki Suzuki $^{a}$ \\ Ryohsuke Kohmoto $^{a}$ Masanori Fukumoto ${ }^{a}$ Kensuke Tajiri ${ }^{a}$

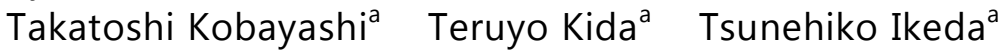 \\ ${ }^{a}$ Department of Ophthalmology, Osaka Medical College, Takatsuki, Japan; \\ ${ }^{b}$ Department of Ophthalmology, Takatsuki Red Cross Hospital, Takatsuki, Japan
}

\section{Keywords}

Rhegmatogenous retinal detachment - Late stage - Endogenous bacterial endophthalmitis .

Vitrectomy

\begin{abstract}
Purpose: To report a case of rhegmatogenous retinal detachment in the late stage, despite the fact that it had previously been halted after intravitreal injection of an antimicrobial agent against endogenous bacterial endophthalmitis (EBE). Case: This study involved a 62-year-old male who had previously been diagnosed with septicemia due to liver abscess and the detection of Klebsiella pneumoniae in a culture of his liver abscess, and who underwent ophthalmic examination after his conjunctival hyperemia had failed to improve. Visual acuity could not be measured due to his general condition being poor and his declining level of consciousness. Slit lamp examination revealed bilateral iritis and cataracts, and the fundus was invisible due to vitreous opacity. Ultrasonic B-mode examination showed subretinal abscess and exudative retinal detachment, leading to the diagnosis of EBE. Vitreous injections of antibiotics were administered to both of his eyes. His right eye became affected by phthisis bulbi, but the condition in his left eye subsided, leaving a scarred lesion near the macula. However, complete retinal detachment occurred in his left eye approximately 10
\end{abstract}


months after the vitreous injection. During vitreous surgery, proliferative membrane formation was observed in the posterior pole area, and an irregular retinal break was detected in the scar margin caused by the traction of the proliferative membrane. After vitreous surgery, the retina was reattached under silicone oil. Conclusion: In cases of EBE, even if the inflammation has previously subsided, strict follow-up examinations are necessary, since complications such as rhegmatogenous retinal detachment may occur at a late stage.

(C) 2017 The Author(s)

Published by S. Karger AG, Basel

\section{Introduction}

Endogenous bacterial endophthalmitis (EBE), a rare disease that accounts for only 2$8 \%$ of the total number of endophthalmitis cases, reportedly occurs due to bacteremia originating from the infected foci of other organs [1], and the prognosis for visual function in cases of EBE is extremely poor. It has been reported that in Europe and the USA, the common basic disease of EBE is infectious endocarditis [2], while in Asia, the common basic disease of EBE is liver/biliary tract infection [3].

Previous studies have reported cases of retinal detachment associated with EBE - many of which occur in the acute phase - and exudative retinal detachment with subretinal abscess [4-9]. Here we report a case in which rhegmatogenous retinal detachment occurred at a late stage, despite the fact that the inflammation had subsided after intravitreal injection of an antimicrobial agent against EBE.

\section{Case Report}

Case

The patient was a 62-year-old male.

\section{Medical History}

After having been diagnosed with disseminated intravascular coagulation by an internist at a nearby medical clinic, the patient was transferred to the Medical Emergency Center at Takatsuki Red Cross Hospital, Takatsuki, Japan, where Klebsiella pneumoniae was detected from a blood/liver abscess culture. His condition was diagnosed as septicemia due to the liver abscess. Thereafter, meningitis and suppurative spondylitis occurred concurrently. Moreover, since the conjunctival hyperemia in both of his eyes did not improve, a medical house call by an ophthalmologist was requested.

\section{Past Medical History}

The patient had a past medical history of alcohol-related hepatitis, liver abscess, and lumbar degenerative spondylosis.

\section{Clinical Findings at the Time of the Patient's Initial Visit}

Upon examination, the patient's visual acuity (VA) could not be measured due to his poor general condition and low level of consciousness. Bilateral ocular conjunctival hyperemia was observed, and an optical examination revealed iritis, posterior iris synechia, and cataracts. The ocular fundus was invisible due to the vitreous opacity, and an ultrasonic B- 
mode examination revealed subretinal abscess and suspected exudative retinal detachment, leading to the diagnosis of EBE.

\section{Treatment Course}

Although the patient's ocular findings were considered to be an indication for vitreous surgery, the operation was deemed difficult to perform due to his poor general condition. Thus, we injected ceftazidime $2.0 \mathrm{mg} / 0.1 \mathrm{~mL}$ into the vitreous cavity of both of his eyes. Concurrently, we administered an intravenous drip treatment of $600 \mathrm{mg}$ linezolid. Following the ceftazidime injections, the intraocular inflammation and exudative retinal detachment in his right eye did not improve and resulted in no light perception; yet the exudative retinal detachment in his left eye was gradually relieved and his corrected VA improved to 0.02 . Thereafter, even though the scar formation in the macular lesion remained in his left eye, the endophthalmitis subsided and the subretinal abscess nearly disappeared. However, the patient revisited our department approximately 10 months later after having become aware of blurred vision in his left eye.

\section{Clinical Findings at the Time of the Patient's Second Visit}

Upon examination, the patient's corrected VA was no light perception OD and 0.01 OS, and his intraocular pressure was $5 \mathrm{~mm} \mathrm{Hg}$ OD and $2 \mathrm{~mm} \mathrm{Hg}$ OS. The patient's right eye was affected by phthisis bulbi. In his left eye, progression of the posterior iris synechia and cataracts were detected (Fig. 1a) and the fundus was difficult to observe. An ultrasonic B-mode examination revealed total retinal detachment (Fig. 1b).

\section{Subsequent Treatment Course}

For treatment, vitreous surgery was performed on the patient's left eye. First, after pars plana lensectomy, the vitreous gel was excised by use of a vitreous cutter. No posterior vitreous detachment occurred, and an artificial posterior vitreous detachment was created from the posterior to the peripheral pole. A proliferative membrane was observed on the temporal side of the scar lesion near the macula, which had been detected before the onset of retinal detachment, and a retinal break had formed at the temporal edge of the scar lesion due to the traction caused by the proliferative membrane (Fig. 2a). Next, the proliferation membrane was removed with vitreous forceps, and the highly viscous subretinal fluid was discharged from the retinal break. After removal of the peripheral vitreous gel, pneumatic retinal replacement was performed, followed by endolaser photocoagulation around the break (Fig. 2b). In order to relieve the residual vitreous traction, a peripheral encircling procedure was performed, and, finally, silicone oil tamponade was indicated. After surgery, the retina was reattached under silicone oil, the corrected VA improved to 0.02 (uncorrectable) (Fig. 3), and the intraocular pressure was approximately $15 \mathrm{~mm} \mathrm{Hg}$ in his left eye.

\section{Discussion}

Exudative retinal detachment (resulting from subretinal abscess formation), traction retinal detachment (caused by epiretinal membranes), and rhegmatogenous retinal detachment (arising from necrotizing retinal breaks) are three types of retinal detachment that are secondary to EBE $[4,5]$. Each of these three types of retinal detachment can occur in the acute phase. However, traction and/or rhegmatogenous retinal detachment can sometimes cause a pathogenesis that can occur even in the late period after the intraocular inflamma- 
tion has subsided. Even at that point, cataracts and epiretinal membrane formation frequently occur as complications in many retinal diseases other than EBE. These clinical findings are thought to be caused by the production of various cytokines released into the vitreous cavity over a long period of time, due to the blood-ocular barrier breakdown induced by endophthalmitis. It is entirely conceivable that due to the fact that the contraction of the epiretinal membrane causes a tangential traction on the retinal necrosis sites, the formation of retinal breaks and rhegmatogenous retinal detachment may occur.

In the present case, a scarred lesion following the retinal necrosis near the macula was observed prior to the onset of retinal detachment, and due to the formation and contraction of the epiretinal membrane around the lesion, its progression to rhegmatogenous retinal detachment seemed to have occurred. Yonekawa et al. [10] reported that among 18 EBE cases that had been halted via intravitreal injections of antibiotics, rhegmatogenous retinal detachment occurred in 3 eyes 1 year or more after treatment.

It has been reported that retinal detachment can occur after intraocular inflammation has subsided, even in endogenous fungal endophthalmitis, which is considered to have a slow progression. In a previous study, Lingappan et al. [4] reported that among 65 eyes treated for EBE, retinal detachment occurred in 17 eyes (26\%) 18 months after treatment. Thus, strict follow-up examinations are necessary as inflammation can persist following endophthalmitis and cause proliferative changes, irrespective of the bacterial and fungal properties.

In regard to the severity of retinal detachment, the extent of proliferative membrane and the presence or absence of posterior vitreous detachment are considered. Once a retinal break forms in a case with a wide-ranging proliferative membrane, it rapidly progresses to proliferative vitreoretinopathy. In a previous study, Torii et al. [11] reported a case of EBE caused by bacterial meningitis which rapidly progressed to proliferative vitreoretinopathy after the inflammation had subsided. In addition, Umazume et al. [12] reported that they had examined the findings obtained during vitreous surgery in 10 cases of EBE. These findings indicated no evidence of posterior vitreous detachment in 8 of the 10 eyes, and showed that the mean age of the patients was 71.2 years. Thus, although the relationship between development of endophthalmitis and progression of posterior vitreous detachment remains unknown, EBE with no posterior vitreous detachment can occur, even in elderly patients.

From the aspect of visual prognosis, it is crucial to note the site where the retinal necrosis or the retinal scar lesions have occurred. In endogenous fungal endophthalmitis, inflammatory lesions often occur in the posterior pole of the fundus, and EBE also can cause scar lesions in the posterior pole. Although EBE is usually unilateral, bilateral cases are not rare; for instance, Wong et al. [13] reported on 32 eyes of 27 patients with EBE, and $22 \%$ of the cases had bilateral involvement.

In conclusion, the findings of this study show that EBE cases should be treated immediately after diagnosis in order to minimize retinal damage. Moreover, even after the inflammation has subsided, strict follow-up examinations are necessary while paying attention to the onset of late complications such as retinal detachment.

\section{Acknowledgements}

The authors wish to thank John Bush for editing the manuscript. 
Kimura et al.: A Case of Rhegmatogenous Retinal Detachment at Late Stage following Endogenous Bacterial Endophthalmitis

\section{Statement of Ethics}

This study was approved by the Ethics Committee of Osaka Medical College.

\section{Disclosure Statement}

There are no conflicts of interest to report for all authors.

\section{References}

1 Jackson TL, Eykyn SJ, Graham EM, Stanford MR: Endogenous bacterial endophthalmitis: a 17-year prospective series and review of 267 reported cases. Surv Ophthalmol 2003;48:403-423.

2 Okada AA, Johnson RP, Liles WC, D'Amico DJ, Baker AS: Endogenous bacterial endophthalmitis. Report of a ten-year retrospective study. Ophthalmology 1994;101:832-838.

3 Wong JS, Chan TK, Lee HM, Chee SP: Endogenous bacterial endophthalmitis: an east Asian experience and a reappraisal of a severe ocular affliction. Ophthalmology 2000;107:1483-1491.

4 Lingappan A, Wykoff CC, Albini TA, Miller D, Pathengay A, Davis JL, Flynn HW Jr: Endogenous funga endophthalmitis: causative organisms, management strategies, and visual acuity outcomes. Am J Ophthalmol 2012;153:162-166.

-5 Foster RE, Rubsamen PE, Joondeph BC, Flynn HW Jr, Smiddy WS: Concurrent endophthalmitis and retinal detachment. Ophthalmology 1994;101:490-498.

-6 Tsai TH, Peng KL: Metastatic endophthalmitis combined with subretinal abscess in a patient with diabetes mellitus - a case report. BMC Ophthalmol 2015;15:105.

7 Liang L, Lin X, Yu A, Lin A, Yuan Z: The clinical analysis of endogenous endophthalmitis. Yan Ke Xue Bao 2004;20:144-148.

-8 De Smet MD, Carlborg EA: Managing severe endophthalmitis with the use of an endoscope. Retina 2005;25:976-980.

-9 Yarng SS, Hsieh CL, Chen TL: Vitrectomy for endogenous Klebsiella pneumoniae endophthalmitis with massive subretinal abscess. Ophthalmic Surg Lasers 1997;28:147-150.

10 Yonekawa Y, Chan RV, Reddy AK, Pieroni CG, Lee TC, Lee S: Early intravitreal treatment of endogenous bacterial endophthalmitis. Clin Exp Ophthalmol 2011;39:771-778.

11 Torii H, Miyata H, Sugisaka E, Ichikawa Y, Shinoda K, Inoue M: Bilateral endophthalmitis in a patient with bacterial meningitis caused by Streptococcus pneumoniae. Ophthalmologica 2008;222:357-359.

12 Umazume K, Suzuki J, Usui Y, Wakabayashi Y, Goto H: Possible relation between lack of posterior vitreous detachment and severe endogenous endophthalmitis. J Ophthalmol 2016;2016:8561379.

13 Wong JS, Chan TK, Lee HM, Chee SP: Endogenous bacterial endophthalmitis: an east Asian experience and a reappraisal of a severe ocular affliction. Ophthalmology 2000;107:1483-1491. 


\section{Case Reports in Ophthalmology}
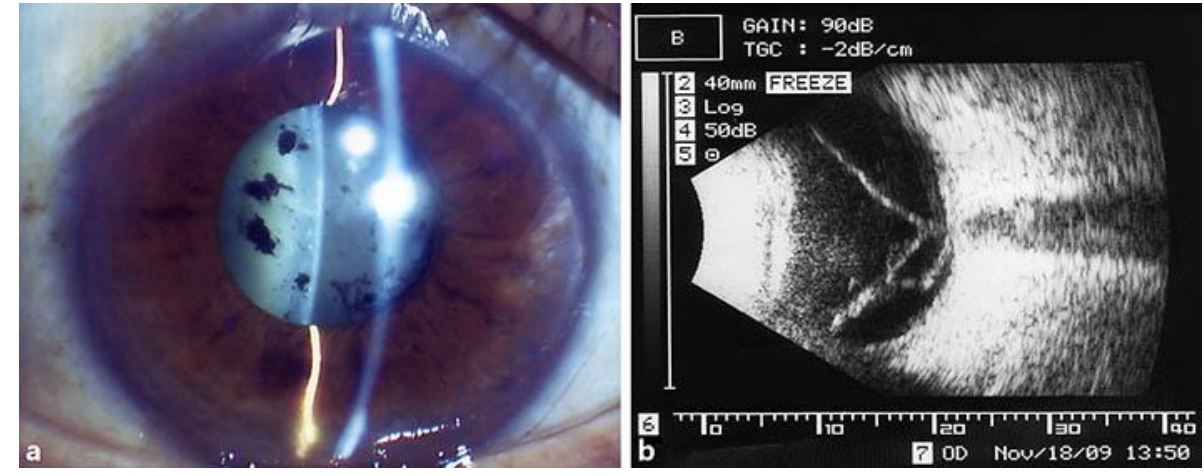

Fig. 1. Preoperative clinical findings. a Slit lamp examination revealed the progression of posterior iris synechia and cataracts. b Ultrasonic B-mode examination showed total retinal detachment.
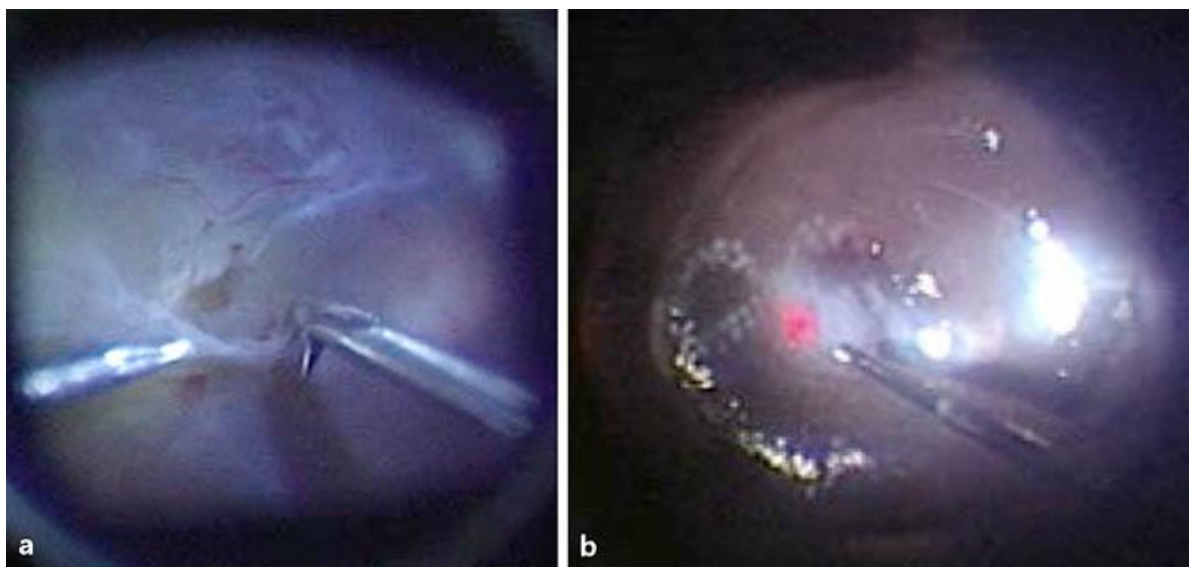

Fig. 2. Intraoperative clinical findings. a A retinal break had formed at the temporal edge of the scar lesion due to the traction caused by the proliferative membrane. b After pneumatic retinal replacement, endolaser photocoagulation around the break was performed. 
Case Reports in
Ophthalmology

Case Rep Ophthalmol 2017;8:334-340 DOI: $10.1159 / 000477160$

(c) 2017 The Author(s). Published by S. Karger AG, Basel www.karger.com/cop

Kimura et al.: A Case of Rhegmatogenous Retinal Detachment at Late Stage following Endogenous Bacterial Endophthalmitis

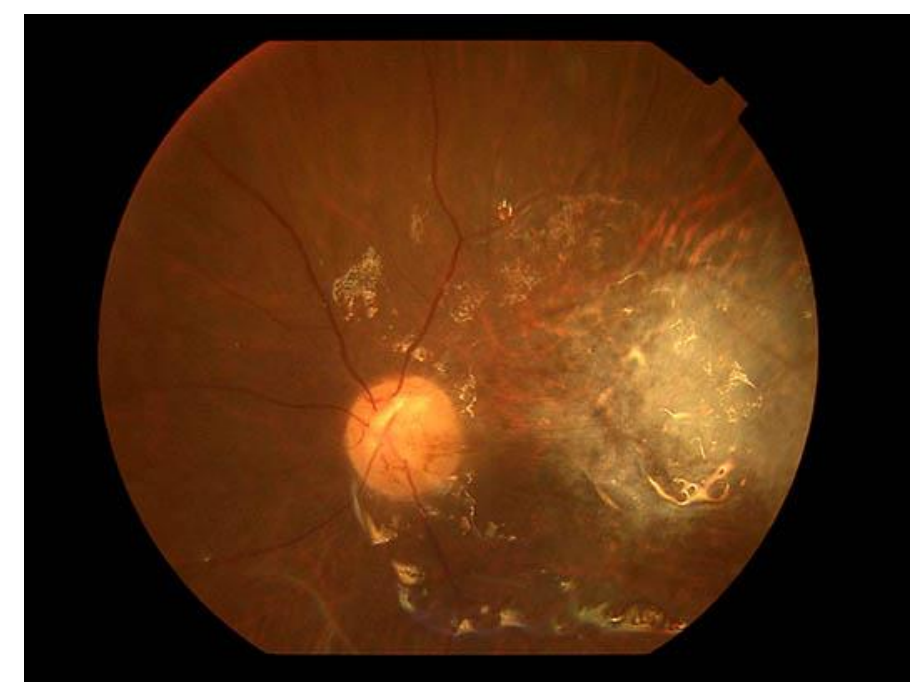

Fig. 3. Postoperative fundus findings. The retina was reattached under silicone oil. 